\title{
REQUIREMENTS FOR NOISE PARAMETER MEASUREMENTS IN SUPERCONDUCTING ELECTRONIC SYSTEMS'
}

\author{
J.M. O'Callaghan, J.B. Beyer
}

University of Wisconsin - Madison

Electrical and Computer Engineering Department

1415 Johnson Dr. Madison, Wl 53706

1. ABSTRACT

General considerations for the measurement of noise parameters in highly mismatched systems are discussed. In particular, the problem of noise characterization of active superconducting microwave devices is addressed. A measurement technique including an error analysis is presented along with current data for a Superconducting Flux Flow Transistor.

\section{2- INTRODUCTION}

Continuing research in superconducting electronics has resulted in the introduction of active microwave devices. These include the VFT (1) and recently the SFFT (2),(3) for high Tc systems. Such devices are typically characterized by very low impedance levels when compared with $50 \Omega$ systems. We are particularly interested in a noise characterization of these devices at microwave frequencies. The SFFT is extremely mismatched and the technique for noise parameter measurement as weil as an error sensitivity analysis is presented.

\section{3- NOISE FIGURE MEASUREMENT METHOD}

Our measurement scheme is based on the HP8970 noise figure meter. This instrument has the capability of internally removing the effects of the noise figure of the measurement system from the one of the device under test (DUT). This correction (see ref. (4), pg. 19) approximates the available gain of the DUT by its insertion gain. Such approximation is appropriate when the device is matched, but large discrepancies occur when this is not satisfied. However, the HP8970 is a very sensitive receiver and can be used in an uncorrected mode. This has given rise to measurement schemes capable of measuring mismatched devices. Such schemes have been applied to microwave and millimeter wave FETs (5), (6).

'This work is partially supported by the Spanish Ministry of Education and Science

\section{1-Basic Noise Equations.}

The noise figure of a two-port is defined as (7):

$$
F=\frac{N_{0}}{G_{0} N_{\mathbf{I}}}
$$

Where $N_{i}, N_{o}$ are respectively the available noise power at the input and output and $G_{a}$ is the available gain of the two port.

When several two-ports are cascaded, their overall noise figure is given by:

$$
F=F_{1}+\frac{F_{2}-1}{G_{1}}+\frac{F_{3}-1}{G_{1} G_{2}}+\cdots
$$

$F_{i}, G_{i}, i=1,2,3 \ldots$ being the noise figure and available gain of the $i$-th two port (with numeration starting from the input side).

Finally, the noise figure of a two port depends on the admittance of the source to which it is connected through the equation (8):

$$
F=F_{\min }+\frac{R_{n}}{G_{8}}\left|Y_{s}-Y_{0}\right|^{2}
$$

Where $F_{\min }, R_{n}, Y_{0}$ are referred as the noise parameters of the two-port and $Y_{S}$ is the source admittance.

\section{2-Measurement of Noise Fioure of Mismatched} Devices

The measurement system used, suggested by J. Mondal (Honeywell Inc.) for highly mismatched DUTs, is shown in Fig. 1 (5). With this setup, the noise source is first connected to the receiver (RCV) and its noise figure is read with the HP8970 meter. The DUT is then inserted and the overall noise figure of the DUT and RCV is read. Since the noise figure of the detection system (DS in Fig.1) remains 


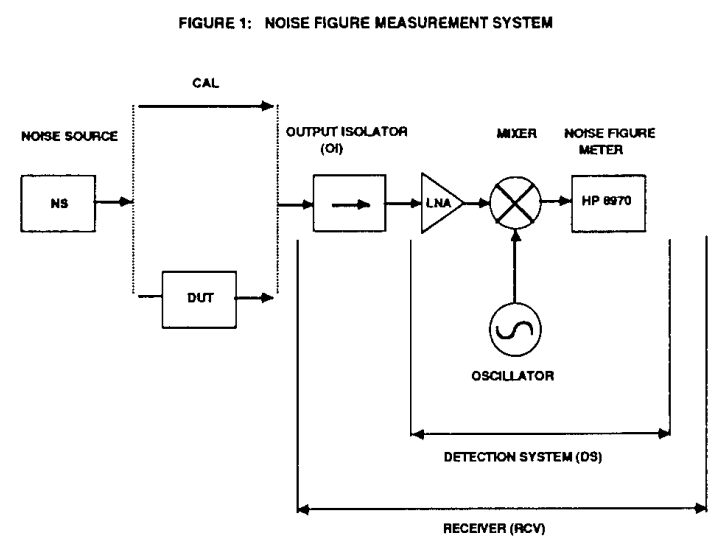

constant in both cases due to the effect of the output isolator (OI), the noise figure of the DUT can be found using Eq. (3.2) and the fact that in passive networks, the noise figure is the inverse of the available gain (8):

$F_{\text {dut }}=F_{\text {rd_ns }}-\frac{F_{\text {rev_ns }}\left(\frac{G_{\text {av_ol_ns }}}{G_{\text {av_ol_dut }}}\right)-1}{G_{\text {av_dut }}}$

where:

$F_{\text {dut }}=$ Noise Figure of the DUT

$F_{r d} n s=$ Uncorrected noise figure of DUT+RCV when connected to the noise source

$F_{\text {rcv_ns }}=$ Uncorrected noise figure of the RCV when connected to the noise source (no DUT inserted)

$\mathrm{G}_{\mathrm{av} \_ \text {oi_ns }}=$ Available gain of the output isolator when connected to the noise source.

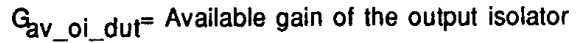
when connected to the DUT.

$G_{a v \_d u t}=$ Available gain of the DUT when connected to the noise source.

Knowledge of the S parameters of the OI and DUT is necessary to calculate the above available gains.

\section{4- NOISE PARAMETER DETERMINATION}

The four noise parameters $\left(F_{\min }, R_{n}, G_{0}, B_{\alpha}\right)$ dictate the dependence of the noise figure of the device with the source admittance through Eq. (3.3). In the approach that we use, the noise parameters are first estimated by fitting Eq.(3.3) to a set of source reflection coefficients that span the Smith Chart (9). Then the source reflection coefficient is set to the predicted optimum for minimum noise $\left(\Gamma_{0}\right)$, and an experimental gradient search of $F_{\min }$ is made. This procedure removes the fitting errors from $F_{\min }$ and $\Gamma_{0}$.

\section{1-Noise Parameter Measurement Scheme}

The experimental setup of Fig. 1 has to be slightly modified to allow for noise parameter measurements (Fig. 2). The DUT block now has to include an input isolator (II), tuner and "device of interest" (DOI). The noise figure of the DOI can be found from that of the DUT and Eq.(3.2). Specifically:

$$
F_{\text {dol }}=F_{\text {dut }} \mathbf{G}_{\text {av_din }}
$$

where DIN stands for "device input network", as indicated in Fig. 2.

FGURE 2. DUT FOR NOISE PARAMETER EXTRACTION

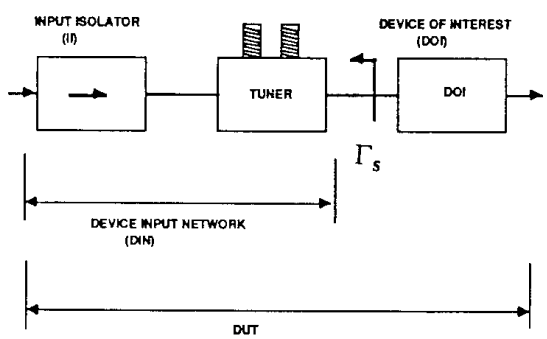

5- ERROR ANALYSIS

As pointed out earlier, $\Gamma_{0}$ is directly measured in the experimental gradient search of $F_{\mathrm{min}}$. Therefore its accuracy depends solely on the capability of the network analyzer used. However, $F_{\min }$ is determined from experimental data through Eqs. (3.4) and (4.1); its ultimate accuracy depends on the errors introduced by both the noise figure meter and the network analyzer.

\section{1-Errors in Fdut}

Equation (3.4) can be simplified by making the following approximations in the available gains involved (8):

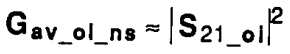

$$
\begin{aligned}
& \mathbf{G}_{\text {av_oi_dut }} \approx\left(1-\left|\Gamma_{\text {out_dut }}\right|^{2}\right)\left|\mathbf{S}_{21 \_ \text {ol }}\right|^{2} \\
& \mathbf{G}_{\text {av_dut }} \approx \frac{\left|\mathbf{S}_{21 \_d u t}\right|^{2}}{\left(1-\left|\Gamma_{\text {out_dut }}\right|^{2}\right)}
\end{aligned}
$$

where the new symbols introduced are defined as: 
$S_{21 \_ \text {oi }}$ Parameter $S_{21}$ of the OI

$S_{21}$ dut: Parameter $S_{21}$ of the DUT

$\Gamma_{\text {out_dut }}$ : Output reflection coefficient of the DUT when connected to the noise source.

Under these assumptions Eq.(3.4) reads:

$F_{\text {dut }} \approx F_{\text {rd_ns }}-\frac{\left(F_{\text {rcv_ns }}+\left|\Gamma_{\text {out_dut }}\right|^{2}-1\right)}{\left|S_{21 \_d u t}\right|^{2}}$

Equation (5.2) agrees within a few percent with the values given by Eq.(3.4), and from it we can conclude that the errors in the measurement of the phases of the $S$ parameters play a secondary role compared to those of their magnitudes, provided that $\left|\Gamma_{\text {out_dut }}\right|$ and $\mid S_{21}$ dut $\mid$ are measured directly.

Taking the proper partial derivatives in Eq.(5.2) and after some algebraic manipulations it can be shown that:

$$
\begin{aligned}
& \frac{\Delta F_{\text {dut }}}{F_{\text {dut }}}=\left(1+\frac{1}{G_{\text {av_dut }} F_{\text {dut }}}\left(\frac{2 F_{\text {rev_ns }}}{1-\left|\Gamma_{\text {out_dut }}\right|^{2}}-1\right)\right) R_{n f}+ \\
& +\frac{1}{G_{\text {av_dut }} F_{\text {dut }}}\left(\frac{\left.\Gamma_{\text {out_dut }}\right|^{2}+F_{\text {rcv_ns }}}{1-\left|\Gamma_{\text {out_dut }}\right|^{2}}-1\right) R_{\text {na }}
\end{aligned}
$$

$R_{n f}$ and $R_{n a}$ being the percent errors introduced by the noise figure meter and the network analyzer respectively, i.e:

$$
R_{n f}=\frac{\Delta F}{F} ; R_{n a}=\frac{\Delta\left(\left|S_{21}\right|^{2}\right)}{\left|S_{21}\right|^{2}}
$$

Equation (5.3) summarizes all the important aspects of the error performance of the measurement system, namely:

- The errors depend on three parameters of the system $\left(F_{r c v \_n s}, R_{n f}\right.$ and $\left.R_{n a}\right)$ and two parameters determined by the device (the product $\mathrm{G}_{\mathrm{av} \_ \text {dut }} \mathrm{F}_{\text {dut }}$ and $\left.\left|\Gamma_{\text {out_dut }}\right|\right)$.

- For a given device, the errors increase as $F_{r c v \_n s}$ $R_{n f}$ and $R_{n a}$ increase.

- For a given system, the errors increase as the product $G_{a v \_d u t} F_{\text {dut }}$ decreases and as $\left|\Gamma_{\text {out_dut }}\right|$ increases.

\section{2-Determination of the system parameters}

The values of $R_{n f}$ and $R_{n a}$ to be used in Eq. (5.3) are derived from the uncertainties (in $\mathrm{dB}$ ) in the noise figure meter and the network analyzer setup, i.e.:

$$
\mathbf{R}_{\mathrm{nf}}=10^{0.1 \mathrm{Rnf}(\mathrm{dB})}-1
$$

for $R_{n f}$ and similarly for $R_{n a}$.

The HP8970 application manual (4) specifies a noise figure uncertainty of $0.25 \mathrm{~dB}$ for frequencies above $1.5 \mathrm{GHz}$. This includes several factors that generate uncertainty when the instrument's standard calibration procedure is used. They include the uncertainty in the matching of the DUT and the second stage correction uncertainty. These factors should not be inculded in $R_{n f}$ when the present calibration method is used, and they have been removed from the noise figure meter uncertainty giving a value of $0.18 \mathrm{~dB}$ for $R_{n f}$.

The uncertainty in $\left|S_{21}\right|^{2}$ is determined by the network analyzer setup. In our case the errors caused by calibration and external cabling dominate those generated internally by the instrument (HP 8753), i.e. its typical uncertainty $( \pm 0.005 \mathrm{~dB})(10)$ is not a liming factor for $R_{\text {na. }}$. A value of about $0.05 \mathrm{~dB}$ has been achieved by minimizing the length of flexible cable and careful connector handling.

The remaining relevant system parameter is $F_{\text {rcv_ns- }}$ Its value is dominated by the noise figure of the external amplifier (see Fig.1). Poor phase stability of the synthesizer can degrade the value of $\mathrm{F}_{\mathrm{rcv}} \mathrm{ns}$. Our system has a noise figure of $2.2 \mathrm{~dB}$ at $3 \mathrm{GHz}$ and uses a GaAs $2-4 \mathrm{GHz}$ amplifier and a HP8673A signal generator.

Equation (5.3) has been plotted as a function of $G_{a v}$ dut $F_{\text {dut }}$ with $\left|\Gamma_{\text {out_dut }}\right|$ as a parameter (see Fig. 3) for the values of $F_{\text {rcv_ns }}, R_{n f}$ and $R_{n a}$ of our system. Note that when the product $G_{a v \_d u t} F_{\text {dut }}$ is high, the noise figure of the DUT dominates the overall noise figure of the setup in Fig.1 and $\Delta F_{\text {dut }} / F_{\text {dut }}$ is given by $R_{n f}$, which is the common horizontal asymptote for all curves in Fig. 3 .

\section{6- ERRORS IN DOI}

Once the noise figure of the DUT is determined, $F_{d o i}$ can be be found through Eq.(4.1). It follows from this equation that the uncertainty in $F_{\text {doi }}$ is given by:

$$
\frac{\Delta F_{\text {dol }}}{F_{\text {dol }}}=\frac{\Delta F_{\text {dut }}}{F_{\text {dut }}}+\frac{\Delta G_{\text {av_dln }}}{G_{\text {av_din }}}
$$

A few algebraic manipulations show that:

$$
\frac{\Delta G_{a v_{-} d i n}}{G_{a v \_d i n}} \approx \frac{\Delta\left(\left|S_{21}\right|^{2}\right)}{\left|S_{21}\right|^{2}}+\frac{1}{\left|S_{21}\right|^{2}} \frac{\left|S_{22}\right|^{2}}{1-\left|S_{22}\right|^{2}} \frac{\Delta\left(\left|S_{22}\right|^{2}\right)}{\left|S_{22}\right|^{2}}
$$



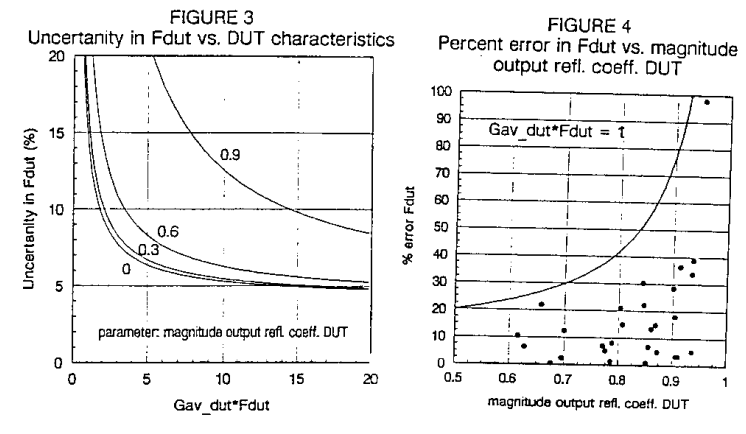

where the s-parameters shown refer to those of the DIN (Fig.2). The first term in Eq.(6.2) is $R_{n a}$ as defined in Eq.(5.4). Similarly $\Delta\left|S_{22}\right|^{2} /\left|S_{22}\right|^{2}$ is a measure of the uncertainty in $\left|S_{22}\right|$. If we assume that it equals $R_{\text {na }}$ and perform a few simplifications, we get:

$$
\frac{\Delta \mathbf{G}_{\mathrm{av} \_d i n}}{\mathbf{G}_{\mathrm{av} \_ \text {din }}} \approx\left[1+\frac{\left|\Gamma_{\mathbf{s}}\right|^{2}}{\left(1-\left|\Gamma_{\mathbf{s}}\right|^{2}\right)^{2}}\right] \mathbf{R}_{\mathbf{n a}}
$$

\section{7- SYSTEM VERIFICATION}

Proper operation of the system can be verified by inserting passive devices as DOI. When this is done, both the DUT and the DOI are passive, and their noise figures $(F)$ are given by the inverse of their available gains $\left(G_{a v}\right)$. In these cases $\Delta F / F$ is given by $\left(1-F^{\star} G_{a v}\right)$.

Figure 4 shows $\Delta F_{\text {dut }} / F_{\text {dut }}$ versus $\left|\Gamma_{\text {out_dut }}\right|$ as calculated by Eq.(5.3) (solid line) versus the relative error found from $G_{a v}$ dut -which is calculated from s parameters(dots in Fig. 4). The latter has been done with four different DOl's and for each of them $\Gamma_{S}$ was adjusted to the set of seven values used in the first step of our noise parameter determination procedure (9),(11).

The same comparison has been made in $F_{\text {doi }}$. It has been found that the uncertainty increase in $F_{\text {doi }}$ with respect that of $F_{\text {dut }}$ is negligible if a good network analyzer calibration is achieved. This is consistent with Eq. (6.3).

Our experience indicates that proper system operation relies heavily on being able to achieve good calibration, particularly for the network analyzer. The value of $R_{\text {na }}$ increases when this is not done, making it difficult to achieve good error performance for high values of $\left|\Gamma_{\text {out_dut }}\right|$.

A verification procedure like the one implied in Fig. 4 is necessary to determine whether or not the calibration should be repeated.

\section{8- MEASUREMENT OF SFFT DEVICE. CURRENT WORK}

The noise figure measurement system described was used in a previous work (11) to determine the noise figure of a SFFT at $3 \mathrm{GHz}$. Since the DOI included the fixture and access cables to the dewar, the resulting $F_{\min }$ should be an upper bound for that of the SFFT itself.

The minimum noise figure was measured at $0.9 \mathrm{~dB}$ for a source reflection coeff. of $\Gamma_{S}=\Gamma_{0}=0.17<-83^{\circ}$. We are currently fabricating more devices in order to asses the repeatability of this value of $F_{\mathrm{min}}$, along with providing reliable error bounds with the verification method presented in this article.

\section{9- ACKNOWLEDGEMENTS}

J.M. O'Callaghan expresses his gratitude to Jyoti Mondal and Vlad Sokolov of Honeywell inc. for valuable noise measurement discussions.

\section{0 - REFERENCES}

(1) D.P.Mc. Ginnis, J.B. Beyer, J.E. Nordman. IEEE-ED Vol. 35 \# 2 Feb. 88. "Vector S-Parameter Measurements of the Superconducting Flux Flow Transistor".

(2) J.S. Martens, G.K.G. Hohenwarter, J.B. Beyer, J.E. Nordman. "Superconducting Thin Film 3 Terminal Devices Made of High Tc and low TC Materials". JAP 65 (10) 15 May 1989.

(3) J.S. Martens, J.B. Beyer, J.E. Nordman. "A Model and an Equivalent Circuit for an Abrikosov Vortex Flow Transistor". to appear in IEEE Trans. on Microwave Superconductivity.

(4) Hewlett Packard. Product Note 8970 A-1. "Applications and Operation of the 8970A Noise Figure Meter". October 1982 .

(5) Jyoti Mondal. Private communication.

(6) Davison,A.C.; Leake,B.W.;Strid E. "Accuracy Improvements in Microwave Noise Parameter Measurements". IEEE Trans. MTT Vol. MTT-37 No.12 December 1989.

(7) F.G. Stremler. "Introduction to Communication Systems. Analysis and Design". Addison Wesley 1982.

(8) Guillermo Gonzalez. "Microwave Transistor Amplifiers. Analysis and Design".Prentice Hall 1984.

(9) J.M. O'Callaghan, J. P. Modal. "A Vector Approach for Noise Parameter Fitting and Selection of Source Admittances". To appear in IEEE Trans. MTT.

(10) Hewlett Packard. Part Number 08753-90022. HP 8753 Network Analyzer. On-Site System Manual. December 1986 .

(11) J.M. O'Caliaghan, J.S. Martens, J.S. Thompson, J.B. Beyer, J.E. Nordman. "Microwave Noise Parameter Measurements of a High Temperature Superconducting Flux Flow Transistor". 1990 Applied Superconductivity Conference. Snowmass Village, Colorado. 